\title{
Sintesis de Ceramicos de ZnO Dopados con Praseodimio y Cobalto
}

\author{
Ana Milena Cruz ${ }^{\mathrm{a}}$ Humar Avila , Jorge Enrique Rodríguez-Páez $z^{\mathrm{a} *}$, \\ Mirian Susana Castro ${ }^{\mathrm{b}}$ \\ ${ }^{\mathrm{a}}$ Grupo CYTEMA, Departamento de Física, Universidad del Cauca \\ Calle 5 No 4 - 70. Popayán - Cauca/Colombia \\ ${ }^{\mathrm{b}}$ Instituto de Insvestigaciones en Ciencia y Tecnología de Materiales (INTEMA) \\ (CONICET-Universidad Nacional de Mar del Plata) \\ Av. J. B. Justo 4302 (B7608FDQ) Mar del Plata, Argentina
}

Received: April 29, 2002; Revised: January 7, 2003

\begin{abstract}
Zinc oxide, $\mathrm{ZnO}$, is a very interesting compound in science and electronic ceramics technology. $\mathrm{ZnO}$ ceramics containing some additives were reported to show varistor action with excellent nonlinearity in current - voltage characteristics. However, investigations were restricted to the $\mathrm{ZnO}-\mathrm{Bi}_{2} \mathrm{O}_{3}$ ceramic system. Other type of varistor, which contains praseodymium oxide, also present nonlinear behaviour. In this work, results obtained in the synthesis $\mathrm{Zn}$-Pr-Co powder are shown. In order to determine and control the several stages of the chemical method, potentiometric titration was used. The solid phase obtained was pressed and sintered.
\end{abstract}

Keywords: zinc oxide, earth rares, powder synthesis, varistor

\section{Introducción}

Las propiedades eléctricas del óxido de cinc, $\mathrm{ZnO}$, se hallan ampliamente estudiadas en la literatura ${ }^{1}$. Al respecto, se ha determinado que cerámicos de $\mathrm{ZnO}$ que contienen algunos aditivos presentan un comportamiento característico corriente-tensión altamente no-óhmico, conocido como efecto varistor ${ }^{2}$. Los varistores se utilizan para proteger líneas de potencia eléctrica y componentes electrónicos contra posibles sobretensiones ${ }^{3,4}$. Al respecto, la función principal del varistor es sensar y limitar la sobretensión transitoria sin que el dispositivo se destruya ${ }^{5}$.

Al presente, existen principalmente dos tipos de varistores cerámicos de $\mathrm{ZnO}$ con alto valor del exponente no-lineal: (a) los cerámicos que contienen óxido de bismuto $^{2-5}$ y (b) los que contienen praseodimio ${ }^{6,7}$ como formadores de barrera en la capa intergranular. De acuerdo a las observaciones microestructurales reportadas en la literatura $^{6-10}$, la capa intergranular del cerámico estaría constituida por un compuesto de praseodimio, sin presencia de átomos de cinc y cobalto, y su espesor sería menor de unos $100 \AA$ A. La resistencia eléctrica de esta capa es baja por lo que el efecto varistor no se puede atribuir a la capa intergranular, sino a la región interfacial entre esta capa y el grano de cinc dopado con cobalto, donde se forma la capa

*e-mail: jnpaez@atenea.ucauca.edu.co de agotamiento ${ }^{6}$. La sinterización de estos cerámicos de cincpraseodimio-cobalto no ocurre en presencia de fase líquida, por el alto punto de fusión de los óxidos de tierras raras, y sólo dos fases se encuentran presentes en el cerámico: granos de $\mathrm{ZnO}$ dopados con cobalto y la fase intergranular constituida principalmente por óxido de praseodimio ${ }^{6-8}$. La ausencia de otras fases incrementa la actividad del área del borde de grano, por donde fluye la corriente eléctrica, aumentando la efectividad del área de la sección transversal del dispositivo y mejorando su comportamiento como protector de sobretensiones.

En la literatura no existen muchos trabajos que traten sobre la síntesis de materia prima ha utilizar en la fabricación de varistores de cinc-praseodimio-cobalto. Normalmente se utiliza la técnica cerámica convencional, mezcla de óxidos, partiendo de $\mathrm{ZnO}$ comercial y de los óxidos de praseodimio, $\operatorname{Pr}_{6} \mathrm{O}_{11}$, y cobalto, $\mathrm{Co}_{3} \mathrm{O}_{3}$, como principales aditivos $^{6-9}$ Esta mezcla se muele en seco, o en húmedo en presencia de un alcohol, y en algunas ocasiones se somete calcinación a $700{ }^{\circ} \mathrm{C}$ durante una hora. Otros métodos de síntesis por ruta química, para introducir los aditivos mencionados en el varistor de $\mathrm{ZnO}$ y distribuirlos uniformemente en su microestructura, se reportan en la literatura ${ }^{11-}$ ${ }^{14}$. Los polvos cerámicos se han obtenido por evaporación de soluciones o suspensiones con una posterior calcina- 
ción ${ }^{11,12}$, combustión rápida en úrea de los nitratos de cinc y de los dopantes ${ }^{13}$ y a través de la precipitación con amoniaco del hidróxido de praseodimio previo a su adición a los otros óxidos ${ }^{14}$. Un trabajo específico sobre la obtención de compuestos de praseodimio y cobalto, utilizando precipitación con amoniaco, fue realizada por Olugbade y colaboradores ${ }^{10}$.

Todos estos trabajos sobre obtención de polvos cerámicos de cinc-praseodimio-cobalto tienen como característica común que describen adecuadamente la preparación pero no ofrecen, como lo indica Matijevic ${ }^{15}$ de una manera general, una base conceptual de principios físicos y químicos que permitan la generalización del proceso y un mayor conocimiento sobre los mecanismos de formación de las partículas. La conformación de una fase sólida, en el seno de una solución, depende de varios parámetros entre los que se destacan: el pH del sistema, la temperatura, la naturaleza de los aniones, la concentración de los reactivos, el método de mezclado, etc ${ }^{16}$. Concretamente el $\mathrm{pH}$ de la suspensión, y los aniones presentes en ella, determinan la naturaleza de los complejos específicos del metal, como soluto, que preceden o dan origen a los núcleos de la fase sólida. Es necesario, por lo tanto, determinar las reacciones que conducen a la formación de la fase sólida y los procesos fisicoquímicos que favorecen tanto la conformación de los núcleos como el mecanismo de crecimiento de los mismos. Este estudio, además de ayudar a establecer y determinar los principios para la construcción de una base conceptual para explicar los procesos de síntesis, permitiría generalizar los procesos y garantizar la reproducibilidad de las características de los polvos cerámicos obtenidos.

En este artículo se reporta la obtención de polvos cerámicos de óxido de cinc-praseodimio y óxido de cincpraseodimio-cobalto utilizando el método de precipitación controlada (MPC). Se determinaron las diferentes etapas del proceso y se analizaron los principales fenómenos fisicoquímicos que ocurren durante su desarrollo. Además se indican resultados preliminares del procesamiento cerámico que permite obtener muestras densificadas de estos sistemas.

\section{Procedimiento Experimental}

\subsection{Síntesis de la Materia Prima}

El polvo cerámico de óxido de cinc que contiene praseodimio y praseodimio-cobalto se obtuvo utilizando el método de precipitación controlada (MPC). El precursor de cinc empleado fue acetato de cinc, $\mathrm{Zn}\left(\mathrm{CH}_{3} \mathrm{COO}\right)_{2}$ (Aldrich). El Zn( $\left.\mathrm{CH}_{3} \mathrm{COO}\right)_{2}$, se disolvió en $200 \mathrm{ml}$ de agua destilada desionizada, sin o con ácido. La solución se agitó a una velocidad de 400 r.p.m. hasta que no se observaron partículas del precursor. Con el objeto de obtener muestras con cinc y praseodimio, se adicionó acetato de praseodimio, $\operatorname{Pr}\left(\mathrm{CH}_{3} \mathrm{COO}\right)_{3} \cdot 3 \mathrm{H}_{2} \mathrm{O}$ (City Chemical Corporation), a la solución anterior en una cantidad adecuada para obtener $0.039,0,088,0,193$ ó $0,25 \%$ en moles de praseodimio en el polvo cerámico final. El sistema se mantuvo siempre en agitación.

Se adicionó hidróxido de amonio, $\mathrm{NH}_{4} \mathrm{OH}$ concentración 15M (Mallinckrodt), para favorecer la hidrólisis de los cationes que existen en el sistema. Para esta adición se empleó un dosificador (Dosimat 774, Metrohm) a una velocidad de $6,67 \times 10^{-4} \mathrm{ml} / \mathrm{s}$. La variación del $\mathrm{pH}$ del sistema se determinó mediante un pH-metro (Metrohm referencia 744). El sistema se llevó a un valor de $\mathrm{pH}$ de 8.5 y al alcanzar este valor se suspendió el suministro de $\mathrm{NH}_{4} \mathrm{OH}$.

La suspensión coloidal obtenida se dejó envejecer durante 42 h y luego se filtró. La fase sólida resultante se redisolvió en etanol y se dejó envejecer por 48 h. Luego, se volvió a filtrar. Este proceso se repitió en dos oportunidades. El sólido obtenido del último filtrado se secó en una estufa, entre $60{ }^{\circ} \mathrm{C}$ y $70{ }^{\circ} \mathrm{C}$, durante dos días. Las muestras sólidas secas se molieron utilizando un mortero de ágata. Este polvo se calcinó a $350{ }^{\circ} \mathrm{C}$ para garantizar que las muestras contuvieran principalmente óxido de cinc y óxido de praseodimio $^{9-10}$. El color del polvo cerámico obtenido fue blanco.

Para obtener los polvos cerámicos de cinc-praseodimiocobalto se procedió de manera similar. El acetato de cobalto, $\mathrm{Co}\left(\mathrm{CH}_{3} \mathrm{COO}\right)_{2} \cdot 4 \mathrm{H}_{2} \mathrm{O}$ (Aldrich), se disolvió en la solución de cinc-praseodimio en una cantidad que permitiera obtener un porcentaje de $1,64,1,84,3,7$ o 5,57\% en moles en el polvo cerámico final. Las cantidades de acetato de cinc, praseodimio y cobalto, se seleccionaron manteniendo constante el contenido de praseodimio, $0,039 \%$ en moles, y se variaron adecuadamente las concentraciones de $\mathrm{Zn}\left(\mathrm{CH}_{3} \mathrm{COO}\right)_{2}$ y $\mathrm{Co}\left(\mathrm{CH}_{3} \mathrm{COO}\right)_{2}$. Los pasos siguientes del proceso se realizaron del mismo modo que en el sistema cinc-praseodimio descrito anteriormente.

Los polvos cerámicos de cinc-praseodimio y cobalto que se obtuvieron presentaban un color rosado debido a la presencia del cobalto.

\subsection{Procesamiento Cerámico}

Los polvos cerámicos obtenidos se prensaron utilizando cargas de 5,09 MPa, $12 \mathrm{MPa}, 19,1 \mathrm{Mpa}$ y 25,4 MPa para obtener pastillas de $2 \mathrm{~mm}$ de espesor y $1 \mathrm{~cm}$ de diámetro. En las muestras prensadas a 5,09 MPa fue necesario adicionar un aglomerante, alcohol polivinílico PVA, con el fin de obtener una mejor compactación de las muestras.

Las pastillas obtenidas se sinterizaron entre $1050{ }^{\circ} \mathrm{C}$ y $1350{ }^{\circ} \mathrm{C}$ durante 2 y $4 \mathrm{~h}$ al aire. La velocidad de calentamiento fue de $5{ }^{\circ} \mathrm{C} / \mathrm{min}$ y el enfriamiento se realizó a la velocidad de enfriamiento del horno. 


\subsection{Caracterización}

Las muestras sinterizadas fueron molidas para caracterizar las fases cristalinas presentes en ellas mediante Difracción de rayos-X, en un difractómetro (Dif5000 Siemens) utilizando radiación $\mathrm{CuK} \alpha$.

\section{Resultados y Discusión.}

\subsection{Obtención de los Polvos Cerámicos.}

En la Fig. 1 se muestran las curvas de valoración potenciométrica correspondientes al sistema cincpraseodimio. La Fig. 1a indica la variación que sufren las curvas de valoración al incrementar la concentración de $\operatorname{Pr}\left(\mathrm{CH}_{3} \mathrm{COO}\right)_{3}$ en la solución inicial sin ácido. En ella se observa que las curvas no experimentan una modificación apreciable al incrementar la concentración inicial del precursor de praseodimio. La región inicial, prácticamente lineal, que presentan las curvas de valoración representa la hidrólisis y posterior condensación de las especies hidrolizadas lo que da origen a los embriones y núcleos de la fase sólida ${ }^{17}$. Este proceso produce un elevado consumo de hidroxilos por el sistema. Estos hidroxilos son empleados para neutralizar los $\mathrm{H}^{+}$que se desprenden durante la hidrólisis de las especies acuo de los cationes ${ }^{17}$ :

$\left[\mathrm{M}\left(\mathrm{OH}_{2}\right)_{\mathrm{N}}\right]^{\mathrm{z+}} \Leftrightarrow\left[\mathrm{M}(\mathrm{OH})_{\mathrm{h}}\left(\mathrm{OH}_{2}\right)_{\mathrm{N}-\mathrm{h}}\right]^{(\mathrm{z}-\mathrm{h})+}+\mathrm{hH}^{+}{ }_{\text {solvatado }}$

y además estos $\mathrm{OH}$ forman "puentes" entre complejos polinucleares que se constituyen durante la adición de la base debido a la presencia de ligandos hidroxo (especie nucleofílica) y acuo, en las especies hidrolizadas, que favorecen las reacciones de condensación por olación (formación de puentes $\mathrm{OH})^{17}$.

Debido a que las curvas de valoración potenciométrica dan información, principalmente de la interfase soluto-solvente, y de los fenómenos que allí ocurren ${ }^{18}$, el comportamiento de las curvas de la Fig. 1a se puede justificar considerando la adsorción de $\operatorname{Pr}^{3+}$, o complejos hidrolizados de praseodimio, en la superficie de las partículas coloidales constituidas mayoritariamente por compuestos de cinc (catión más fácilmente hidrolizable). Mediante estudios realizados paralelamente a este trabajo, y en concordancia con datos de la bibliografía ${ }^{10}$, se estableció que el hidróxido de praseodimio hidratado es el principal compuesto que precipita en sistemas de $\mathrm{Pr}\left(\mathrm{CH}_{3} \mathrm{COO}\right)_{3}-\mathrm{NH}_{4} \mathrm{OH}$.

A continuación se presentan las reacciones superficiales de adsorción que se proponen pueden ocurrir en el sistema en estudio tomando como base las reacciones generales que se plantean en la literatura ${ }^{18}$ :

$$
\equiv \mathrm{S}-\mathrm{OH}+\mathrm{Pr}^{3+}+\mathrm{H}_{2} \mathrm{O} \Leftrightarrow \equiv \mathrm{S}-\mathrm{OPrOH}^{+}+2 \mathrm{H}^{+}
$$

(a)

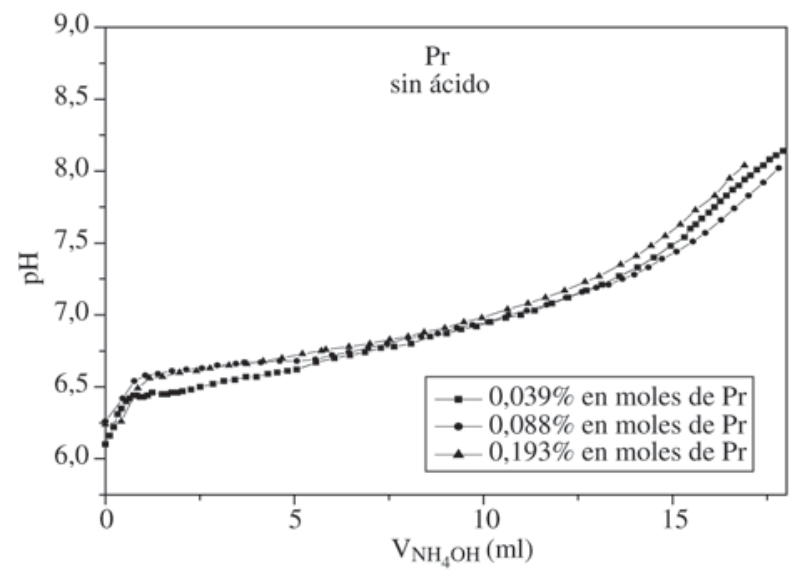

(b)

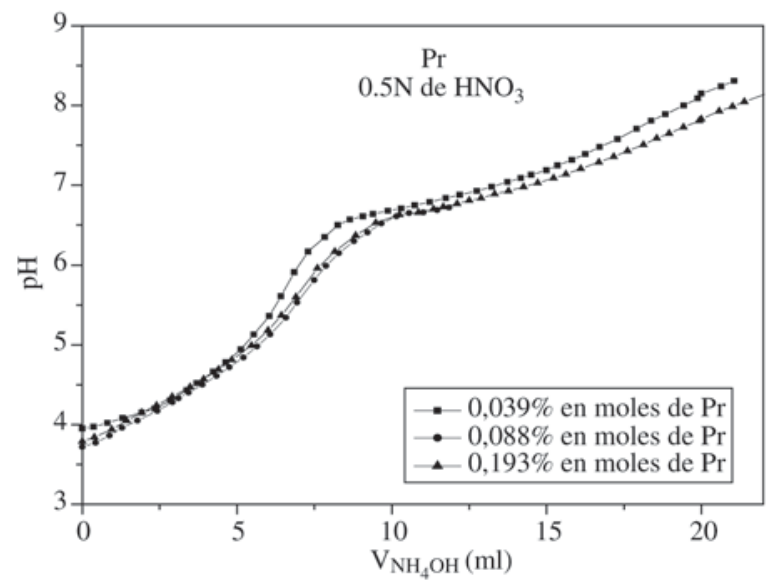

Figura 1. Curvas de valoración potenciométrica obtenidas para el sistema acuoso de cinc - praseodimio sin ácido (a) y con ácido (b).

Esta reacción ocurre si el catión no está hidrolizado, $\equiv \mathrm{S}-\mathrm{OH}$ representa un grupo hidroxilo superficial, pero si en el sistema existen especies hidrolizadas del tipo $\left[\mathrm{Pr}\left(\mathrm{H}_{2} \mathrm{O}\right)_{\mathrm{x}}\right]^{3+},{ }^{19} \mathrm{y} \operatorname{Pr}(\mathrm{OH})^{2+},{ }^{20}, \mathrm{o}\left[\mathrm{Pr}(\mathrm{OH})\left(\mathrm{H}_{2} \mathrm{O}\right)_{\mathrm{x}-1}\right]^{2+}$, puede ocurrir un proceso de fisisorción en la superficie ${ }^{17}$ :

$\left.\left.\equiv \mathrm{S}-\mathrm{OH}+\left[\mathrm{Pr}(\mathrm{OH})\left(\mathrm{H}_{2} \mathrm{O}\right)_{\mathrm{x}-1}\right)\right]^{2+} \Leftrightarrow \equiv \mathrm{S}(\mathrm{OH})_{\mathrm{m}},\left[\mathrm{Pr}(\mathrm{OH})\left(\mathrm{H}_{2} \mathrm{O}\right)_{\mathrm{x}-1}\right)\right]^{2+}+\mathrm{mH}_{2} \mathrm{O}$

Un proceso equivalente puede suceder si se considera la estequiometría del intercambio de protones, lo que involucra la hidrólisis de los complejos en la superficie ${ }^{17}$ :

$\left.\equiv \mathrm{S}-\mathrm{OH}+\left[\mathrm{Pr}\left(\mathrm{OH}_{2}\right)_{\mathrm{x}}\right)\right]^{3+} \Leftrightarrow \equiv \mathrm{S}(\mathrm{O}),\left[\mathrm{Pr}\left(\mathrm{OH}_{2}\right)_{\mathrm{x}-1}\right]^{2+}+\mathrm{H}_{3} \mathrm{O}^{+}$

En las reacciones anteriores se considera que el Pr tiene una valencia +3 . 
Según la literatura ${ }^{17}$ pueden ocurrir dos procesos dependiendo del pH del sistema. Puede suceder que la hidrólisis del catión ocurra y aumente la posibilidad de su adsorción, ecuación (3), o que la hidrólisis sea consecuencia de la adsorción, ecuación (4), Tanto a través de la ecuación (2) como de la ecuación (4), el proceso superficial lleva a un incremento de la concentración de $\mathrm{H}^{+}$en el sistema, lo que exige un mayor suministro de $\mathrm{NH}_{4} \mathrm{OH}$, o sea $\mathrm{OH}^{-}$, para neutralizar protones. Este es el comportamiento que presenta la curva de valoración potenciométrica correspondiente a la menor concentración de $\operatorname{Pr}\left(\mathrm{CH}_{3} \mathrm{COO}\right)_{3}$, e indica la gran importancia que tiene la adsorción superficial de praseodimio, o de complejos hidratados de praseodimio, en este caso.

Para las otras concentraciones de praseodimio el comportamiento de las curvas de valoración es similar a la descrita, por lo tanto deben presentarsen en estos sistemas los mismos fenómenos y procesos descritos a través de la ecuaciones (2-4). Por otro lado, la existencia de una mayor cantidad de especies hidrolizadas, del tipo $\left.\left[\mathrm{Pr}(\mathrm{OH})\left(\mathrm{H}_{2} \mathrm{O}\right)_{\mathrm{x}-1}\right)\right]^{2+}$, favorece el proceso de condensación y la posterior conformación del precipitado de hidróxido de praseodimio hidratado ${ }^{10}$.

En la Fig. 1b se ilustra el efecto de la adición de $\mathrm{HNO}_{3}$ al sistema. En ella se puede observar que independientemente de la concentración inicial de $\operatorname{Pr}\left(\mathrm{CH}_{3} \mathrm{COO}\right)_{3}$ las curvas de valoración potenciométrica obtenidas son todas similares. La principal diferencia con las curvas de la Fig. 1a es la presencia de una zona inicial donde se presenta una apreciable variación del $\mathrm{pH}$ del sistema, y que debe corresponder a la neutralización de las especies ácidas presentes en el sistema por el $\mathrm{NH}_{4} \mathrm{OH}$ adicionado. A continuación se encuentra la zona de alto consumo de $\mathrm{OH}^{-}$, es decir, donde ocurren los procesos de hidrólisis y condensación de las especies de cinc y praseodimio dando origen a los embriones y núcleos de la fase sólida. Si se considera la existencia de praseodimio en el sistema, el $\mathrm{NO}_{3}^{-}$favorecería el incremento de su número de coordinación pudiendo alcanzar valores de 10 o más ${ }^{19}$.

En la Fig. 2 se muestran las curvas de valoración correspondientes a un sistema acuoso, sin y con ácido, que contiene la misma cantidad de praseodimio y diferentes concentraciones de cobalto. En la Fig. 2a Se observa una apreciable variación de las curvas al modificar la concentración de $\mathrm{Co}\left(\mathrm{CH}_{3} \mathrm{COO}\right)_{2}$. La curva que corresponde a $1,64 \%$ en moles tiene un comportamiento similar al de las curvas de la Fig. 1a. Para concentraciones mayores, el valor de $\mathrm{pH}$ inicial disminuye y las curvas presentan tramos lineales con distintos valores de pendiente. Inicialmente se presenta una disminución del pH y luego comienza a incrementarse lentamente con la adición del $\mathrm{NH}_{4} \mathrm{OH}$. A un valor de $\mathrm{pH} \sim 6,5$ se presenta el primer cambio apreciable de pendiente.

El comportamiento de estas curvas se puede justificar si

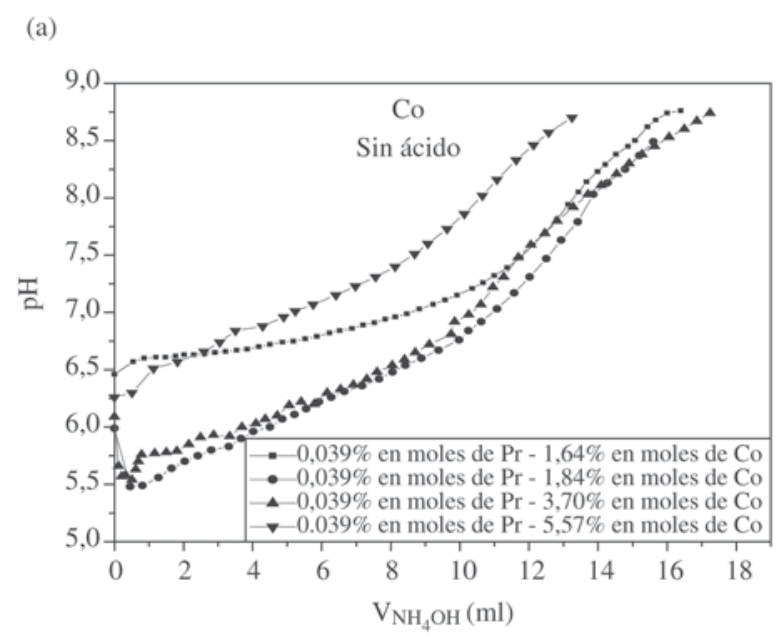

(b)

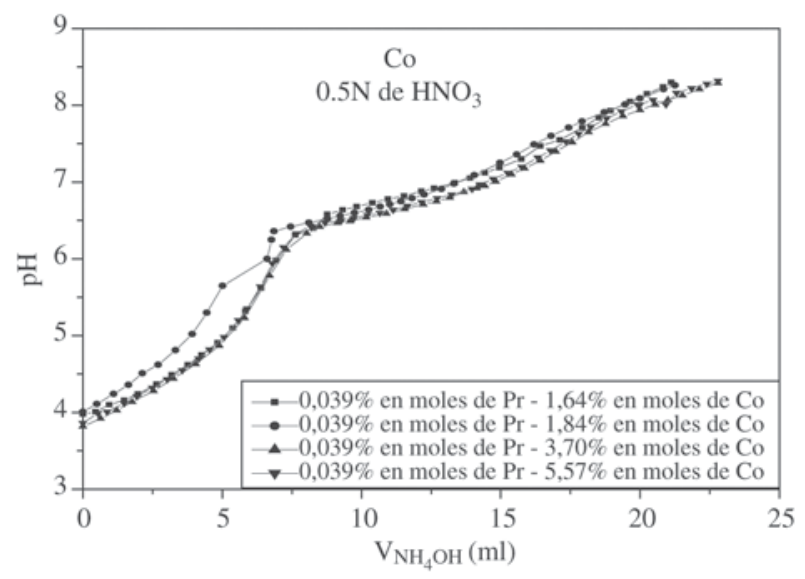

Figura 2. Curvas de valoración potenciométrica obtenidas para el sistema acuoso de cinc - praseodimio - cobalto, manteniendo el contenido de praseodimio constante - $0,039 \%$ en moles, sin ácido (a) y con ácido (b).

se tiene en cuenta la quimisorción del cobalto en la superficie de las partículas coloidales constituidas principalmente por compuestos de cinc. Debido a que el cobalto se hidroliza fácilmente, al igual que otros metales de transición, éste tiene una gran afinidad por las superficies de los coloides y su adsorción puede ocurrir en contra de las fuerzas electrostáticas ${ }^{17}$. La adsorción de este catión depende fuertemente del $\mathrm{pH}$ del sistema y puede ocurrir en un rango de pH entre 1 o 2 unidades, la velocidad de adsorción (grado de saturación de la superficie) en este rango de $\mathrm{pH}$ varía de 0 a $100 \%$ tal como sucede en la adsorción de Co (II) por goetita, $\alpha-\mathrm{FeOOH}^{21}$. Estudios paralelos a este trabajo, realizados en nuestro laboratorio, y datos de la literatura ${ }^{10-17,20}$, indican que si únicamente existe $\mathrm{Co}^{2+} \mathrm{en} \mathrm{la}$ solución se forma el complejo $\left.\left[\mathrm{Co}(\mathrm{OH})\left(\mathrm{OH}_{2}\right)_{5}\right)\right]^{+}$, a un $\mathrm{pH} 7$, y que a un $\mathrm{pH}$ de 8 debe precipitar inicialmente el 
inestable $\alpha-\mathrm{Co}(\mathrm{OH})_{2}$, que presenta un color verde azulado, para transformarse luego en el $\beta$ - $\mathrm{Co}(\mathrm{OH})_{2}$ que presenta un color rosado. Por tanto, teniendo en cuenta el esquema general del proceso de fisisorción, sobre la superficie de los coloides, debería ocurrir la siguiente reacción:

$\left.\left.\equiv \mathrm{S}-\mathrm{OH}+\left[\mathrm{Co}(\mathrm{OH})_{\mathrm{n}}\left(\mathrm{OH}_{2}\right)_{6-\mathrm{n}}\right)\right]^{(2-\mathrm{n})+} \Leftrightarrow \equiv \mathrm{S}(\mathrm{OH})_{\mathrm{m}},\left[\mathrm{Co}(\mathrm{OH})_{\mathrm{n}}\left(\mathrm{OH}_{2}\right)_{6-\mathrm{n}}\right)\right]^{(2-\mathrm{n})+}+\mathrm{mH}_{2} \mathrm{O}$

En cambio, si se adsorbe el ión hexa-acuo de cobalto, como sucede en el sistema $\mathrm{Co}(\mathrm{II})-(\alpha-\mathrm{FeOOH})^{17}$, se tendría que:

$\left.\left.\equiv \mathrm{S}-\mathrm{OH}+\left[\mathrm{Co}\left(\mathrm{OH}_{2}\right)_{6}\right)\right]^{2+} \Leftrightarrow \equiv \mathrm{S}(\mathrm{O}),\left[\mathrm{Co}\left(\mathrm{OH}_{2}\right)_{6}\right)\right]^{+}+\mathrm{H}_{3} \mathrm{O}^{+}$

Esta reacción justificaria, en parte, la disminución del $\mathrm{pH}$ al incrementar la concentración inicial de $\mathrm{Co}\left(\mathrm{CH}_{3} \mathrm{COO}\right)_{2}$, principalmente para $\mathrm{pH}<6,5$.

Debido a que el $\mathrm{Co}(\mathrm{OH})_{2}$ precipita a $\sim \mathrm{pH} 8$, es muy probable que para $\mathrm{pH}>8$ exista sobre la superficie de los coloides $\mathrm{Co}(\mathrm{OH})_{2}$ y $\mathrm{CoO}(\mathrm{OH})$, como ocurre en otros sistemas $^{17}$. Lo anterior indica que es posible que la hidrólisis y precipitación del cobalto ocurra en la solución, y que las partículas precipitadas posteriormente se fisisorban sobre las partículas coloidales que existen en el sistema. Este comportamiento sería diferente al que se presentaría para $\mathrm{pH}<6.5$ donde la hidrólisis del Co(II) sería inducida por grupos superficiales más que por la acidez de la solución, como se indica en la ecuación (6), los hidroxilos superficiales desempeñarían el papel de ligandos hidrolizantes y la formación de complejos en la esfera interna favorecería la quimisorción del cobalto.

No hay que olvidar, que además de los procesos superficiales anteriormente indicados, las curvas de la Fig. 2a contienen información de la hidrólisis y condensación del cinc y de la adsorción del praseodimio (dado el bajo contenido en el sistema de esta tierra rara, Fig. 1b). Lo anterior se torna más complejo si se considera que en sales amónicas, y en medio neutro, precipita una sal básica de cobalto de color azul ${ }^{22}$.

En un trabajo paralelo que se está realizando, se ha observado que el precipitado es soluble en exceso de amoníaco y produce una solución pardo amarillenta que debe contener el complejo $\mathrm{Co}\left(\mathrm{NH}_{3}\right)_{6}{ }_{6}^{2+}$. En cambio, si hay abundancia de cobalto, se forman copos de color verde.

En la Fig. $2 \mathrm{~b}$ se presentan las curvas de valoración que se obtienen para sistemas con $\mathrm{HNO}_{3}$ que contienen igual cantidad de praseodimio, y diferente contenido de cobalto y cinc. En ellas se observa que el comportamiento de las curvas es muy similar independiente de las concentraciones. Para comprender mejor lo que sucede con el cobalto en un medio básico, y conocer qué posibles especies y compuestos de cobalto existen, se ha realizado un trabajo paralelo tomando la misma cantidad de $\mathrm{Co}\left(\mathrm{CH}_{3} \mathrm{COO}\right)_{2}$ y disolviéndola en el mis- mo volumen de la solución acuosa acidificada a temperatura ambiente. Se adicionó $\mathrm{NH}_{4} \mathrm{OH}$ y se fue observando la variación de las tonalidades del sistema. Inicialmente la solución adquirió un color rojo indicando la presencia de $\mathrm{Co}^{2+}$ y $\mathrm{Co}(\mathrm{OH})_{2}$. En medio básico, tanto el $\mathrm{Co}^{2+}$ como el $\mathrm{Co}(\mathrm{OH})_{2}$ se pueden oxidar, aún por el oxígeno del medio ambiente, y los productos de la oxidación que se obtienen son: hidróxido cobáltico, $\mathrm{Co}(\mathrm{OH})_{3}$, de color pardo oscuro, u óxidos de un color similar, $\mathrm{Co}_{2} \mathrm{O}_{3}$ o una mezcla de $\mathrm{Co}$ (II) y $\mathrm{Co}$ (III) $\left(\mathrm{Co}_{3} \mathrm{O}_{4}\right)$ más o menos hidratados ${ }^{22}$. El paso de la solución de un color rojo a pardo oscuro no es instantáneo, en la región de transición ácido-básico se observó un color anaranjado-marrón. Actualmente se realizan estudios de espectroscopia UV-visible de estas soluciones.

Por otro lado, el polvo cerámico de cinc-praseodimiocobalto obtenido después de lavarlo con etanol, y secar la suspensión coloidal resultante del proceso, presenta un co-

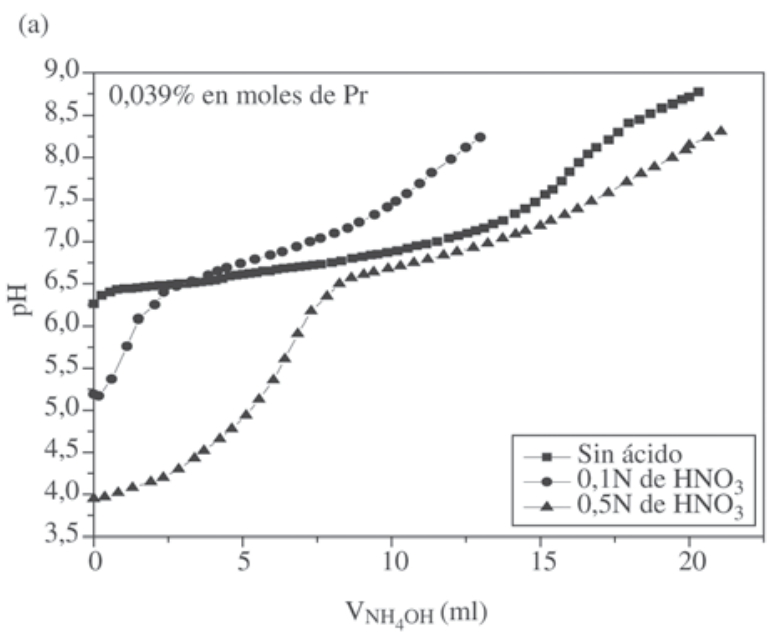

(b)

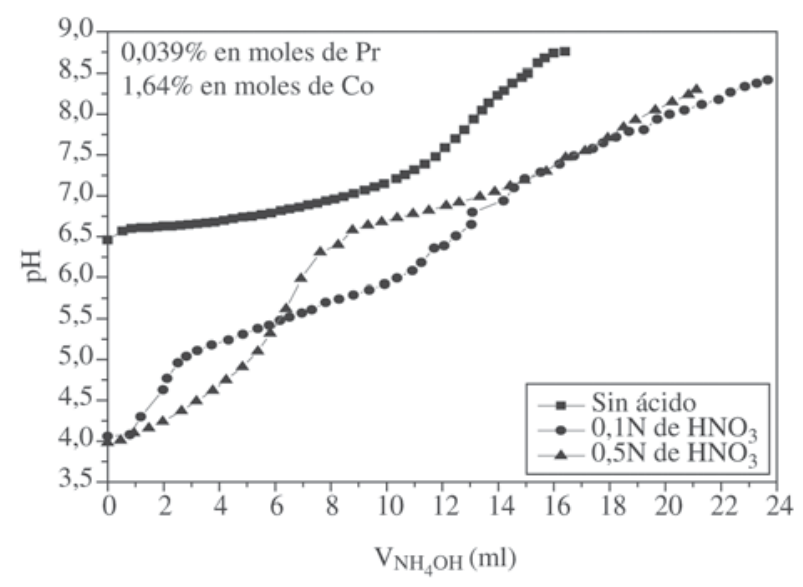

Figura 3. Efecto del contenido de $\mathrm{HNO}_{3}$ sobre las curvas de valoración potenciométrica correspondientes a (a) un sistema acuoso de cinc - praseodimio y (b) un sistema acuoso cinc praseodimio - cobalto. 


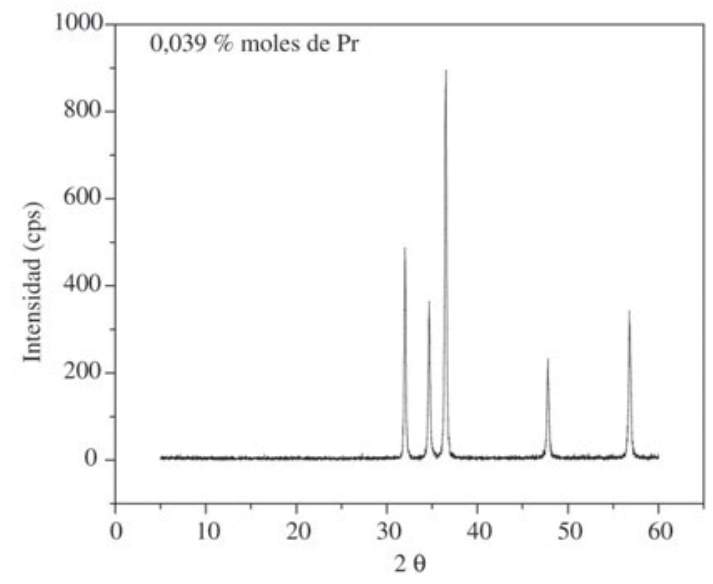

Figura 4. Difractograma de rayos $\mathrm{x}$ de una muestra que contiene $0,039 \%$ en moles de Pr sinterizada a $1300{ }^{\circ} \mathrm{C}$ durante $2 \mathrm{~h}$.

lor rosado que indica la presencia de $\mathrm{Co}(\mathrm{OH})_{2}$ y $\mathrm{CoO}(\mathrm{OH})$, compuestos que pueden estar fisorbidos sobre la superficie de las partículas tal como se indicó anteriormente.

En la Fig. 3 se muestra el efecto del $\mathrm{HNO}_{3}$, adicionado en diferentes concentraciones, sobre los sistemas cincpraseodimio, Fig. 3a, y cinc-praseodimio-cobalto, Fig. 3 b. La variación más relevante de las curvas de valoración potenciométrica, con el aumento de la concentración de ácido, es la aparición e incremento de la zona de neutralización.

\subsection{Obtención de Muestras Densificadas.}

Los polvos cerámicos de cinc-praseodimio y cincpraseodimio-cobalto fueron inicialmente prensados a diferentes valores de presión, y luego sinterizados a temperaturas entre los $1050{ }^{\circ} \mathrm{C}$ y los $1350{ }^{\circ} \mathrm{C}$ durante $2 \mathrm{y} 4 \mathrm{~h}$. Con el fin de determinar las fases cristalinas presentes en el sistema cinc - praseodimio durante la sinterización, se utilizó difracción de rayos-X para caracterizar la muestra que contiene $0,039 \%$ en moles de Pr tratada térmicamente a $1300{ }^{\circ} \mathrm{C}$, Fig. 4. Como era de esperar, la única fase cristalina presente es la cincita. El praseodimio debe ubicarse en la capa intergranular que se forma en los bordes de grano, tal como se indica en la literatura ${ }^{6-8}$. Como el óxido de praseodimio está disperso, y su concentración es muy baja, éste no es detectado por esta técnica de caracterización. De acuerdo a trabajos previos ${ }^{7}$, es de esperar que la fase intergranular sea $\mathrm{Pr}_{2} \mathrm{O}_{3}$, que se formaría de manera directa de la transformación del hidróxido de praseodimio hidratado, presente en el sistema, o durante el proceso de sinterización a través de la siguiente reacción:

$$
\mathrm{Pr}_{6} \mathrm{O}_{11} \rightarrow 3 \mathrm{Pr}_{2} \mathrm{O}_{3}+\mathrm{O}_{2}
$$

La fase $\operatorname{Pr}_{2} \mathrm{O}_{3}$ en la matriz de $\mathrm{ZnO}$ permanece estable
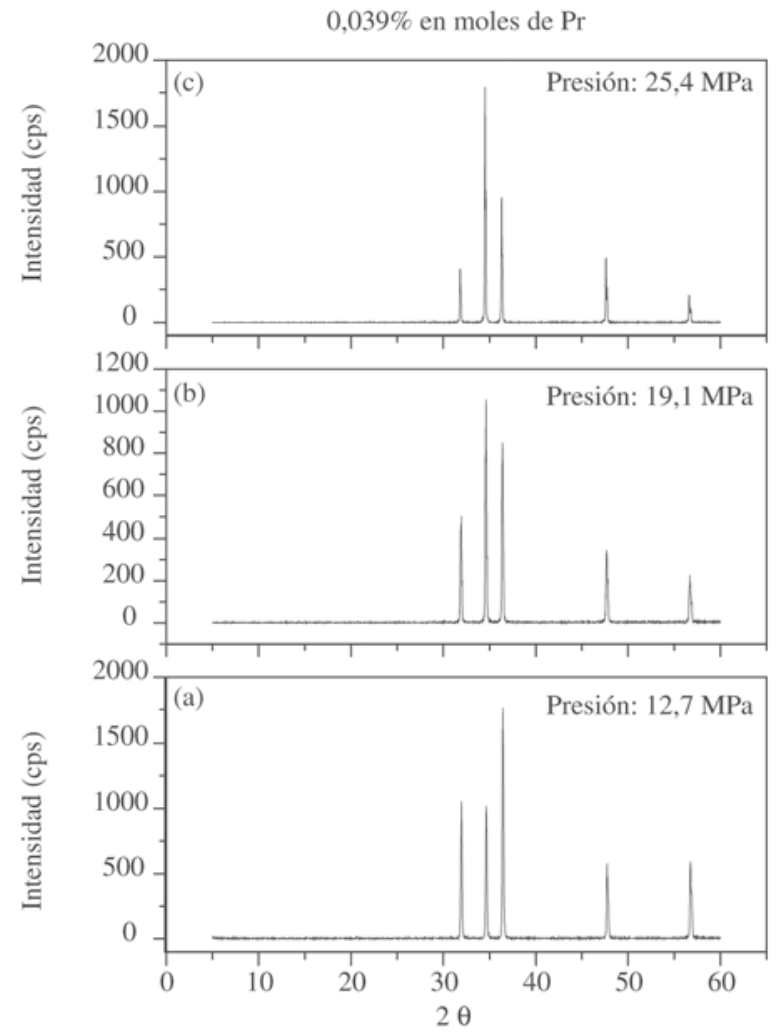

Figura 5. Difractogramas de rayos- $X$ de muestras que contienen $0,039 \%$ en moles de Pr sometidas a diferentes presiones durante el conformado y sinterizadas a $1200{ }^{\circ} \mathrm{C}$ durante $2 \mathrm{~h}$.

debido a que la matriz se comporta como un medio reductor en el que los átomos de cinc intersticiales actúan como agentes reductores durante el proceso de sinterización, a través de la siguiente reacción:

$$
2 \mathrm{Zn}+\mathrm{Pr}_{6} \mathrm{O}_{11} \rightarrow 2 \mathrm{ZnO}+3 \mathrm{Pr}_{2} \mathrm{O}_{3}
$$

Esta ecuación muestra la reacción global ya que realmente la reducción ocurre en varias etapas.

En la Fig. 5 se indica el efecto del prensado sobre las muestras. Los difractogramas corresponden a muestras sometidas a diferentes presiones durante el prensado, y luego sinterizadas a $1200{ }^{\circ} \mathrm{C}$ durante $2 \mathrm{~h}$ (en aire). Como se puede observar el pico con índices de Miller (101) se va destacando cada vez más frente a los picos (100) y (001). Esto indica que se presenta una especie de "texturación" de las muestras al incrementar la presión del prensado; se favorece una cierta dirección y por tanto la anisotropía del material.

\section{Conclusiones}

Considerando los resultados obtenidos, y el análisis realizado, en el presente trabajo, se puede concluir lo siguiente:

1. Mediante el ensayo de valoración potenciométrica, uti- 
lizado para controlar la síntesis de polvos cerámicos de $\mathrm{ZnO}$ $-\mathrm{Pr}_{2} \mathrm{O}_{3}-\mathrm{Co}_{3} \mathrm{O}_{4}$, es posible determinar las diferentes etapas del proceso y las modificaciones que en él se presentan al variar algunos de los parámetros involucrados en el mismo. En soluciones acuosas sin ácido se presentan tres regiones principales que están relacionadas con los fenómenos de formación de embriones y núcleos de fase sólida y con el crecimiento de estos últimos. En sistemas que contienen ácido nítrico aparece una región adicional que está relacionada con el proceso de neutralización del sistema.

2. La explicación de los fenómenos que ocurren durante la formación de la fase sólida en el seno de la solución, se puede abordar a partir de las reacciones que pueden ocurrir en la interfase sólido - líquido y que dan origen a complejos superficiales. Esta propuesta se basa en los procesos de hidrólisis y condensación como un esquema general a considerar. Es posible que la hidrólisis del catión preceda y aumente la posibilidad de su adsorción o que la hidrólisis sea una consecuencia de la adsorción y que dependa del valor de $\mathrm{pH}$ del sistema. Las especies hidrolizadas son las que posteriormente se condensarán, a través de procesos de olación y oxolación, para dar origen a las especies polinucleares, los oligómeros y/o polímeros y a partir de éstos a los embriones $\mathrm{y}$, posteriormente, a los núcleos de la fase sólida.

3. Para el sistema cinc - praseodimio, independientemente de la concentración de praseodimio, los procesos de adsorción superficial sobre las partículas coloidales son muy importantes. Al incrementarse la concentración, la precipitación de hidróxido de praseodimio hidratado en el seno de la solución es también importante.

4. Cuando se adiciona cobalto al sistema, dado que el Co se hidroliza fácilmente, éste se adsorbe dada su gran afinidad por las superficies de los coloides, tal como se ejemplifica en el sistema Co(II) - goetita ${ }^{21}$. Es muy probable que para valores de $\mathrm{pH}$ mayores que 8 existan sobre los coloides $\mathrm{Co}(\mathrm{OH})_{2}$ y $\mathrm{CoO}(\mathrm{OH})$. Hay que realizar estudios adicionales para verificar la naturaleza de estos compuestos superficiales de cobalto.

\section{Agradecimientos}

Este trabajo fue financiado a través del proyecto COLCIENCIAS № 1103-05-605-93 (RC 191-94). Agradecemos a la red CyTED VIIIF de Materiales Electrocerámicos la ayuda económica que le brindo a la Sra. Ana M. Cruz para la estancia que realizó en el INTEMA - Universidad de Mar del Plata - Argentina.

\section{Referencias}

1. Heiland, G.; Mollwo, E.; Stockman, F. en Solid State Physics, eds. F. Seitz and Turnbull, Academic Press, New
York. Vol.8, p.191, 1959.

2. Matsuoka, M. Jpn. J. Appl. Phys., v.10, p. 736, 1971.

3. Eda, K. IEEE Electrical Insulation Magazine, v. 5, n 6 , p. $28,1989$.

4. Gupta, T.K. J. Am. Ceram. Soc., v. 73, n. 7, p. 1817, 1990.

5. Clarke, D.R. J. Am. Ceram. Soc., v. 82, n. 3, p. 485, 1999.

6. Mukae, K.; Tsuda, K.; Nagasawa, I. Jpn. J. Appl. Phys., v. 16, n. 8, p. 1361, 1977.

7. Mukae, K. Am. Ceram. Soc. Bull., v. 66, n. 9, p. 1329, 1987.

8. Mukae, K.; Nagasawa, J. "Effect of praseodymium oxide and donor concentration in the grain boundary region of $\mathrm{ZnO}$ varistors", en Grain Boundary Phenomena in Electronic Ceramics, Advances in Ceramics, Volume 1. The American Ceramic Society, Inc. Columbus Ohio, p. 331, 1981.

9. Fan, J.; Freer, R. "Microstructures and no-ohmic characteristics of $\operatorname{Pr}_{6} \mathrm{O}_{11}$ doped, zinc oxide-bismuth oxide ceramic varistors" en Euroceramics II, Proceedings of Second European Ceramic Society, eds. G. Ziegler, H. Hausner, 11-14 septiembre/Augsburg, p. 2055, 1991.

10. Olugbade, E.A.; Trontelj, M. "Synthesis and Characterization of fine particles of $\mathrm{Pr}$ - Co Oxides", en Euroceramics II, Proceedings of Second European Ceramic Society, editores G. Ziegler, H. Hausner, 1114 septiembre/Augsburg, p. 2067, 1991.

11. Varma, H.K.; Kumar, K.P.; Warrier, K.G.K.; Damodaran, A.D. J. Mat. Sci. Lett., v. 8, p. 974, 1989.

12. Vipin, P.M.; Sanjaynath, V.V.; Varma, H.K.; Warrier, K.G.K.; Damodaran, A.D. Br. Ceram. Trans. J., v. 89, p. 87, 1990.

13. Vipin, P.M.; Sanjaynath, V.V.; Varma, H.K.; Warrier, K.G.K.; Domodaran, A.D. J. Eur. Ceram. Soc., v. 5, p. 233, 1989.

14. Alles, A.B. "Grain Boundary acceptor states in electronic ceramics”, Ph.D Thesis, Alfred University, Alfred- New York, pp. 85-86, 1990.

15. Matijevic, E. Chem. Mater., v. 5, p. 412, 1993.

16. Matijevic, E. Progr. Colloid \& Polymer Sci., v. 61, p. 24, 1976.

17. Jolivet, J.P. Metal oxide chemistry and synthesis, John Wiley \& Sons, Ltd., 2000.

18. Stumm, Chemistry of the Solid - Water interfase, John Wiley \&. Sons, Inc., 1992.

19. Greenwood, N.N.; Earnshaw, A. Chemistry of the elements, Butter- Worth/Heinemann, 1995.

20. Baes Jr., C.F.; Mesmer, R.E. The hydrolysis of cations, John Wiley \&. Sons Inc., 1976.

21. Skhenk, C.V.; Dillard, J.G.; Murray, J.W. J. Colloid Interface Sci., v.95, p. 398, 1983.

22. Martí, F.B.; Conde, F.L.; Jimeno, S.A.; Méndez, J.H. Química Analítica, Editorial Paraninfo, decimoquinta edición, 1994. 